Review Article

\title{
Cancer Surveillance in Healthy Carriers of Germline Pathogenic Variants in BRCA1/2: A Review of Secondary Prevention Guidelines
}

\author{
Boudewijn Dullens $\left(\mathbb{D},{ }^{1,2}\right.$ Robin de Putter $\left(\mathbb{D},{ }^{3}\right.$ Matteo Lambertini $\left(\mathbb{D},{ }^{4,5}\right.$ Angela Toss $(\mathbb{D}),{ }^{6}$ \\ Sileny Han, ${ }^{2,7}$ Els Van Nieuwenhuysen, ${ }^{2,7}$ Toon Van Gorp, ${ }^{2,7}$ Adriaan Vanderstichele, ${ }^{2,7}$ \\ Chantal Van Ongeval, ${ }^{2,8}$ Machteld Keupers ${ }^{(D)}{ }^{2,8}$ Renate Prevos, ${ }^{2,8}$ Valerie Celis, ${ }^{2,8}$ \\ Jeroen Dekervel, ${ }^{9}$ Wouter Everaerts $\mathbb{D}^{1},{ }^{10}$ Hans Wildiers, ${ }^{1,2}$ Ines Nevelsteen $\left(\mathbb{D},{ }^{2,11}\right.$ \\ Patrick Neven, ${ }^{2,7}$ Dirk Timmerman $(\mathbb{D})^{7}$ Ann Smeets $\mathbb{D i D}^{2,11}$ Ellen Denayer ${ }^{(D)}{ }^{2,12}$ \\ Griet Van Buggenhout, ${ }^{2,12}$ Eric Legius $\left.\mathbb{D}\right)^{2,12}$ and Kevin Punie $\mathbb{D}^{1,2}$ \\ ${ }^{1}$ Department of General Medical Oncology, University Hospitals Leuven, Leuven, Belgium \\ ${ }^{2}$ Multidisciplinary Breast Centre, UZ-KU Leuven Cancer Institute (LKI), University Hospitals Leuven, Leuven, Belgium \\ ${ }^{3}$ Department of Medical Genetics, Ghent University Hospital, Ghent, Belgium \\ ${ }^{4}$ Department of Medical Oncology, U.O.C Clinica di Oncologia Médica, IRCCS Ospedale Policlinico San Martino, Genova, Italy \\ ${ }^{5}$ Department of Internal Medicine and Medical Specialties (DiMI), School of Medicine, University of Genova, Genova, Italy \\ ${ }^{6}$ Department of Oncology and Hematology, University Hospital of Modena, Modena, Italy \\ ${ }^{7}$ Department of Gynecology and Obstetrics, University Hospitals Leuven, Leuven, Belgium \\ ${ }^{8}$ Department of Radiology, University Hospitals Leuven, Leuven, Belgium \\ ${ }^{9}$ Digestive Oncology, Department of Gastroenterology, University Hospitals Leuven, Leuven, Belgium \\ ${ }^{10}$ Department of Urology, University Hospitals Leuven, Leuven, Belgium \\ ${ }^{11}$ Department of Surgical Oncology, University Hospitals Leuven, Leuven, Belgium \\ ${ }^{12}$ Department of Human Genetics, KU Leuven, Leuven, Belgium
}

Correspondence should be addressed to Kevin Punie; kevin.punie@uzleuven.be

Received 6 March 2020; Accepted 30 May 2020; Published 20 June 2020

Academic Editor: Jorg Kleeff

Copyright (c) 2020 Boudewijn Dullens et al. This is an open access article distributed under the Creative Commons Attribution License, which permits unrestricted use, distribution, and reproduction in any medium, provided the original work is properly cited.

Germline pathogenic alterations in the breast cancer susceptibility genes 1 (BRCA1) and 2 (BRCA2) are the most prevalent causes of hereditary breast and ovarian cancer. The increasing trend in proportion of cancer patients undergoing genetic testing, followed by predictive testing in families of new index patients, results in a significant increase of healthy germline $B R C A 1 / 2$ mutation carriers who are at increased risk for breast, ovarian, and other BRCA-related cancers. This review aims to give an overview of available screening guidelines for female and male carriers of pathogenic or likely pathogenic germline BRCA1/2 variants per cancer type, incorporating malignancies that are more or less recently well correlated with $B R C A 1 / 2$. We selected guidelines from national/international organizations and/or professional associations that were published or updated between January 1, 2015, and February 1, 2020. In total, 12 guidelines were included. This review reveals several significant discordances between the different guidelines. Optimal surveillance strategies depend on accurate age-specific cancer risk estimates, which are not reliably available for all $B R C A$-related cancers. Up-to-date national or international consensus guidelines are of utmost importance to harmonize counseling and proposed surveillance strategies for $B R C A 1 / 2$ carriers. 


\section{Introduction}

Germline pathogenic alterations in the breast cancer susceptibility genes 1 (BRCA1) and 2 (BRCA2) are the most prevalent causes of hereditary breast and ovarian cancer (HBOC). Family studies and segregation analyses have estimated carrier rates of pathogenic and likely pathogenic $B R C A 1$ or BRCA2 alterations in a mixed western population between 1 in 200 and 1 in 1500 persons with most estimates towards the lower end of the range $[1,2]$. In some populations like Ashkenazi Jews, founder effects are observed with carrier frequencies up to $1 \%$ or more [3]. Better knowledge of the implications of $B R C A$ alterations in cancer treatment led to higher awareness among patients and physicians. Together with improved availability of genetic testing, this has led to lower testing thresholds and more germline diagnostic tests, resulting in an increase of cancer patients with known germline pathogenic variants in $B R C A 1 / 2$. Predictive testing in families of new index patients leads to a further increase of healthy carriers with germline alterations correlated with $B R C A 1 / 2$ and other monogenetic causes of HBOC [4].

There are several implications for carriers of (likely) pathogenic variants in $B R C A 1 / 2$. Besides the increased cancer risks and the identified prognostic and predictive implications in BRCA-related breast, ovarian, pancreatic, and prostate cancers, the autosomal dominant inheritance pattern has important implications for the children and relatives of mutation carriers [5, 6]. Known female and male carriers of pathogenic or likely pathogenic variants who plan to conceive should be counseled about options of prenatal and preimplantation genetic diagnosis [7].

The elevated cancer risks extend beyond breast and ovarian cancer. There is clear evidence for an increased risk for prostate and pancreatic cancer. The risk for other cancers such as stomach, colorectal, and endometrial cancer and melanoma might also be elevated to some extent, and some guidelines give recommendations for these possible associations, while for other reported supposed correlations, none of the guidelines give specific recommendations (e.g., cervical cancer) $[8,9]$. There are important uncertainties and differences in strength of evidence and differential effects for BRCA1 and BRCA2 with regard to these and other possible additional cancer risks. Lifetime risks have not been reliably estimated for all these correlations (Table 1). Given the burden of cancer risks and surveillance for germline carriers of a hereditary cancer syndrome, appropriate counseling about primary and secondary prevention strategies is a crucial factor in the care for these individuals. Several national and international guidelines and algorithms for surveillance of $B R C A$-related cancers exist. With this review, we aim to give an overview and comparison of available screening guidelines for BRCA-related cancers for female and male carriers of pathogenic or likely pathogenic germline $B R C A 1 / 2$ variants per cancer type, incorporating malignancies where a correlation with $B R C A 1 / 2$ is more or less recently well demonstrated.

\section{Methods}

We selected articles for our review by Medline search and additional web-based search of the national and international organizations and/or professional associations for guidelines that reported recommendations on secondary prevention in female and/or male carriers of pathogenic or likely pathogenic germline $B R C A 1 / 2$ variants. Only guidelines published or updated between January 1, 2015, and February 1, 2020, were eligible for inclusion in this review. The review is limited to recommendations available in English, French, or Dutch. Guidelines that did not provide clear information about the starting age of surveillance or about the recommended screening modality were excluded. We retrieved 12 guidelines that met our criteria.

\section{Cancer Surveillance Guidelines in Germline BRCA1/2 Mutation Carriers}

3.1. Breast Cancer. Germline pathogenic variants in BRCA1/ 2 are highly penetrant for breast cancer. The incidence of breast cancer in female $B R C A 1 / 2$ carriers increases rapidly in early adulthood. The breast cancer risk increases between 30 and 40 years in BRCA1, but the higher penetrance of BRCA2 at later ages has been confirmed reaching an absolute cumulative risk between 60 and $80 \%$ at age 80 years for both $B R C A 1$ and $B R C A 2$. The risk of contralateral breast cancer is estimated at $40 \%$ for BRCA1 carriers and $25 \%$ for $B R C A 2$ carriers at 20 years after the first breast cancer diagnosis [10].

The high lifetime risk of breast cancer in female $B R C A$ carriers makes the discussion of primary prevention strategies (lifestyle modifications, chemoprevention, and riskreducing surgery) important. Regarding chemoprevention, only limited data exist on the preventive benefit of tamoxifen in $B R C A 1 / 2$ mutation carriers. In addition, there is some concern about the safety of tamoxifen regarding endometrial cancer risk. Moreover, there is discordance as to whether $B R C A 1$ carriers, who are more prone to estrogen receptor negative breast cancer, benefit as much from this chemoprevention approach as BRCA2 carriers [22, 23]. Several trials investigating new chemoprevention approaches in $B R C A$ carriers are ongoing [24].

Risk-reducing mastectomy (RRM) has been shown to be a very effective breast cancer primary prevention option $[25,26]$. Breast cancer after RRM in BRCA carriers has been reported, but the absolute risk is very low and none of the guidelines propose imaging surveillance after RRM [25, 27]. A cohort study has shown improved overall and breast cancer-specific mortality rates in BRCA1 mutation carriers, while for $B R C A 2$, survival rates were not significantly different after a median follow-up for 10.3 years [28]. In clinical practice, for the vast majority of women opting for RRM, mortality reduction is not the dominant driver in the decision process [29]. The option of RRM should be discussed with female carriers of (likely) pathogenic germline mutations in BRCA1/2. However, risk-reducing surgery should never be recommended as the only option to address the high breast cancer risk, and the advantages and 
TABLE 1: Overview of lifetime cancer risks in carriers of germline BRCA1/2 (likely) pathogenic variants.

\begin{tabular}{|c|c|c|c|}
\hline \multirow{2}{*}{ Type of malignancy } & \multicolumn{3}{|c|}{ Lifetime risk of malignancy } \\
\hline & General population (\%) & BRCA1 (\%) & BRCA2 (\%) \\
\hline Breast, female $[10,11]$ & 12 & 72 & 69 \\
\hline Breast, male [11-13] & 0.1 & 1.2 & $6.8-8.4$ \\
\hline Ovarian $[10,11]$ & $1-2$ & 44 & 17 \\
\hline Pancreatic $^{\ddagger}[14-16]$ & 0.5 & $1-3$ & $2-7$ \\
\hline Prostate $^{\ddagger}[11,17,18]$ & 6 (by age 65 ) & 8.6 (by age 65 ) & 15 (by age 65) \\
\hline Colorectal $^{\ddagger}[11,19]$ & 4-5 & Possibly elevated & $¥$ \\
\hline Endometrial $^{*}[11,20]$ & 3 & Possibly elevated & $¥$ \\
\hline Melanoma ${ }^{\ddagger}[11,21]$ & $2-3$ & $¥$ & Possibly elevated \\
\hline
\end{tabular}

${ }^{\ddagger}$ Lifetime risks not estimated, extrapolated from odds ratios/standardized incidence ratios. ${ }^{¥}$ Insufficient or inconsistent data about possible association with increased risk.

disadvantages of this option and other primary or secondary prevention strategies should be extensively discussed.

Although male breast cancer is a rare disease in the general population, with a lifetime risk of $0.1 \%$ accounting for less than $1 \%$ of all cancers in men and about $1 \%$ of all breast cancers, the cumulative incidence is significantly increased in male $B R C A 1 / 2$ carriers and is estimated at $1 \%$ in $B R C A 1$ carriers and $7-8 \%$ in BRCA2 carriers $[12,13,30]$.

Breast cancer screening in germline mutation carriers is correlated with an increased rate of stage 0 or stage 1 breast cancer, and there is limited data about survival benefit $[31,32]$. There are several guidelines and recommendations for breast cancer surveillance in germline $B R C A$ mutation carriers. A schematic overview of guidelines for female carriers is shown in Figure 1. The majority of guidelines address screening approaches for female and male carriers and discuss recommendations on breast awareness, clinical examination, mammography, and magnetic resonance imaging (MRI), but guidance on the use of digital breast tomosynthesis (DBT) and ultrasound is often not specified. There is concern that the exposure to diagnostic radiation at young age may be associated with an increased risk of breast cancer in BRCA carriers [33]. Moreover, the decision as to whether or not to undergo a RRM is often not made at the recommended starting age for breast cancer screening. Therefore, the starting age of mammography in female carriers is an important aspect of the surveillance guidelines. Some guidelines advise annual screening procedures, while the concern about interval cancers in these high-risk patients leads to semiannual alternating schedules in other recommendations [31]. The option of DBT is mentioned in some guidelines based on the superior sensitivity and specificity compared to standard mammography; however, there is no data on the use in BRCA mutation carriers who undergo MRI screening [34]. In a recent study among 1444 averagerisk women aged 40-70 with heterogeneously dense or extremely dense breast, the invasive cancer detection rate was significantly higher for MRI compared to DBT, and no invasive cancer was identified by DBT alone [35].

\subsubsection{European Society for Medical Oncology Guidelines.} The clinical practice guidelines for cancer prevention and screening in $B R C A$ mutation carriers from the European
Society for Medical Oncology (ESMO) were published in 2016 [36]. For female carriers of pathogenic BRCA variants, breast awareness and clinical breast examination are recommended every 6-12 months from the age of 25 or 10 years before the youngest breast cancer diagnosis in the family, whichever occurs first. Annual MRI is recommended from the age of 25, with the addition of annual mammography from the age of 30 . The decision to introduce mammography before the age of 40 should take into consideration the increased breast density at younger ages and the availability of annual screening MRI. In women $\leq 30$ years, breast ultrasound can be considered in case MRI is unavailable. Ultrasound can also be considered in addition to mammography at all ages and as an alternative when MRI is not available. Upper age limit or other conditions where screening should be discontinued are not described for female carriers. After RRM, routine surveillance is not recommended but should be considered in patients who have undergone nipple-sparing mastectomy. Male carriers should be advised to undergo annual clinical breast examination by a physician from age 30 onwards. Routine annual breast imaging among male carriers is not recommended.

\subsubsection{National Comprehensive Cancer Network Guidelines.} The last version from the clinical practice guidelines in genetic high-risk assessment for breast, ovarian, and pancreatic cancer of the National Comprehensive Cancer Network (NCCN) dates from December 2019 [37]. With regard to breast cancer surveillance in women, breast awareness is recommended starting at age 18 and clinical breast exam every 6-12 months from age 25 . Between age 25 and 29, annual breast MRI with contrast is recommended. Starting age should be individualized based on family history if a breast cancer was diagnosed in a relative before age 30. When MRI is unavailable, annual mammogram with consideration of DBT is recommended. Between ages 30 and75, both annual MRI with contrast and annual mammogram with consideration of DBT are recommended. In carriers $>75$ years, management should be considered on an individual basis. Criteria for high-quality breast MRI include availability of experienced breast MRI radiologists, a dedicated breast coil, the ability to perform MRI-guided biopsies, and regional availability. Breast MRI 


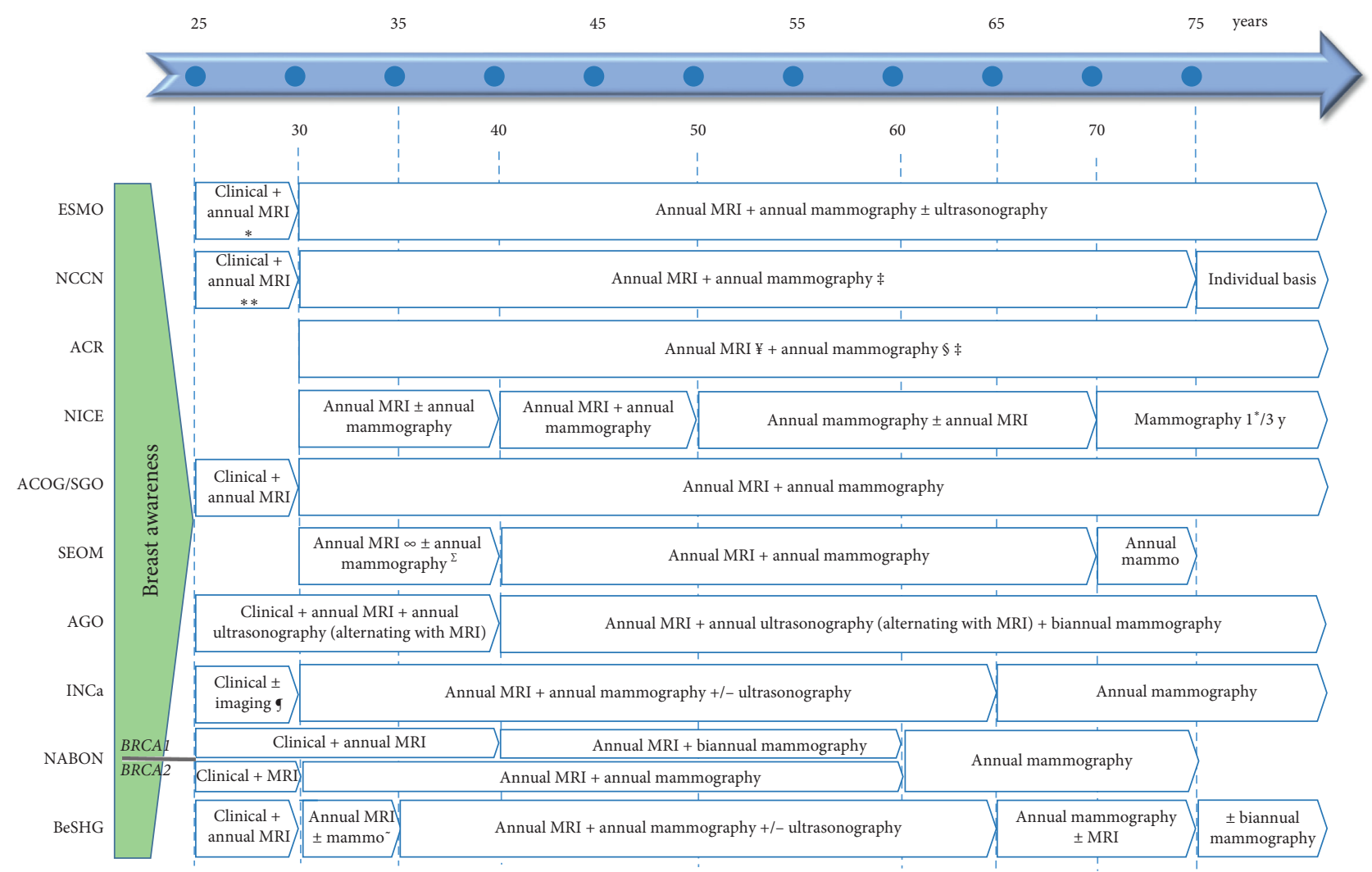

FIGURE 1: Schematic overview of surveillance guidelines for breast cancer in asymptomatic female carriers of (likely) pathogenic BRCA1/2 variants. MRI: magnetic resonance imaging; ESMO: European Society for Medical Oncology; NCCN: National Comprehensive Cancer Network; ACR: American College of Radiology; NICE: National Institute for Health and Care Excellence; ACOG: American College of Obstetricians and Gynecologists; SGO: Society of Gynecologic Oncology; SEOM: Sociedad Espanola de Oncologia Médica; AGO: Arbeitsgemeinschaft Gynäkologische Onkologie; INCa: Institut National du Cancer; NABON: Nationaal Borstkanker Overleg Nederland; BeSHG: Belgian Society of Human Genetics. *Or starting 10 years earlier than youngest breast cancer diagnosis in the family. ${ }^{* *}$ Or individualized based on family history if a breast cancer diagnosis is present before age 30 . ${ }^{\ddagger}$ Or starting $5-10$ years earlier than the youngest breast cancer diagnosis in the family. ${ }^{\S}$ Starting 10 years before the youngest breast cancer diagnosis in the family, but not before 30 . ${ }^{\ddagger}$ Considering breast tomography. ${ }^{\infty}$ Or starting earlier if there is a family history of breast cancer before 30 years. ${ }^{\Sigma}$ Discussing delaying mammography until 40 years with $B R C A 1$ carriers who undergo annual MRI screening. ${ }^{\circ}$ Considering imaging in case of early breast cancer diagnosis in the family. Mammography at age 30, annual mammography from 30 onwards in case of microcalcifications.

is preferably performed on days $7-15$ of a menstrual cycle in premenopausal women.

Male carriers of (likely) pathogenic variants in $B R C A$ are recommended to undergo annual clinical breast examination and undergo training in self-examination with monthly practice starting from age 35 onwards. Regularly scheduled mammography is not recommended in male $B R C A$ carriers.

3.1.3. American College of Radiology. The publication of the Appropriateness Criteria ${ }^{\circledR}$ for breast cancer screening from the American College of Radiology (ACR) dates from 2017 [34]. Recommendations are limited to the radiological imaging procedures, and guidelines for breast cancer screening in women with a $B R C A$ gene mutation are similar to the recommendations for women with a history of chest irradiation between 10 and 30 years of age and women with $\geq 20 \%$ lifetime breast cancer risk. Annual mammography is recommended starting 10 years earlier than the affected relative at the time of diagnosis, but not before 30 years. The superior sensitivity and specificity of DBT over planar mammography are described, and the advantages seem to be most pronounced in women with higher breast density, in women under age 50 , and in carriers with spiculated masses and asymmetries. Since in the majority of situations standard two-dimensional images are obtained in addition to the DBT images, the radiation dose is increased compared to standard mammography. However, virtual planar images created from the tomographic data set could replace the need for a $2 \mathrm{D}$ correlative view in the near future. Surveillance with annual breast MRI (with and without contrast) is recommended in addition to mammography. For the starting age of MRI screening in BRCA carriers, ACR refers to the American Cancer Society Guidelines for breast screening with MRI as an adjunct to mammography. The recommended starting age is 30 years for the majority of women, or 5 to 10 years before the earliest breast cancer diagnosis in the family. The starting age should be based on shared decision making, considering individual preferences and 
circumstances. Screening with breast MRI should be continued as long as the woman is in good health.

\subsubsection{National Institute for Health and Care Excellence} Guidelines. The clinical guidelines on familial breast cancer by the National Institute for Health and Care Excellence (NICE) were originally published in 2013, but the online version was verified as up-to-date in November 2019 [38]. All carriers should be informed about breast awareness. Annual mammography should be considered in female carriers aged 30-39 and recommended aged 40-69, while patients $\geq 70$ years should be offered mammography every three years as part of the population screening program. Mammographic surveillance should never be offered for patients $<30$ years.

Annual MRI surveillance should be offered to female carriers aged 30-49 years and can be considered between 50 and 69 years in case of dense breast pattern but should not be offered to BRCA carriers $<30$ years.

The NICE guidelines state that ultrasound surveillance should not be routinely offered but could be considered when MRI is not possible or when results of mammography or MRI are difficult to interpret. No recommendations are made for male carriers.

The guidelines on breast cancer screening from the London Cancer Alliance (published in 2013 and updated in 2016, [39]) and the Institute of Cancer Research protocol for $B R C A$ mutation carriers $(2015,[40])$ are concordant with the NICE guidelines. The latter specifies that no breast surveillance is recommended for male carriers.

\subsubsection{American College of Obstetricians and Gynecologists/} Society of Gynecologic Oncology. The HBOC clinical management guidelines from the committee on practice bulletins from the American College of Obstetricians and Gynecologists (ACOG) and the committee on genetics from the Society of Gynecologic Oncology (SGO) were last reviewed in 2017 [41]. For woman aged 25-29, recommended surveillance consists of clinical breast examination every $6-12$ months in combination with annual radiographic screening (preferably MRI with contrast). For women $\geq 30$ years, annual mammography and annual MRI with contrast are recommended, often alternating every 6 months. There are no specific statements regarding the use of ultrasonography, or about age limits or male carriers, in these guidelines.

3.1.6. Spanish Society of Medical Oncology. The clinical guidelines in HBOC of the hereditary cancer working group from the Spanish Society of Medical Oncology (Sociedad Espanola de Oncologia Médica, SEOM) were revised in 2019 [42]. Annual breast MRI should be proposed between 30 and 70 years, or earlier in case of family history of breast cancer before 30 years. Addition of annual mammogram should be considered from 30 years onwards and recommended between 40 and 75 years. Delaying mammography until 40 years should be discussed for BRCA1 carriers who undergo annual MRI screening.
When MRI is unavailable, screening with mammography and ultrasound is advised between 30 and 75 years. For male BRCA carriers, the SEOM guidelines advise that screening mammography should be considered only in the presence of gynecomastia.

3.1.7. German Society for Gynecological Oncology. The proposed surveillance program of the German Society for Gynecological Oncology (Arbeitsgemeinschaft Gynäkologische Onkologie, AGO) is available in the latest version of the AGO breast guidelines which were last revised in 2019 [43]. Clinical breast examination is recommended semiannually for female carriers from age 25 onwards. Starting age for annual breast MRI is 25 years. Annual ultrasonography is recommended in interval between the MRI examinations from age 25 onwards. Biannual mammography is recommended starting at age 40 . In upper age limit, other conditions where screening should be discontinued and recommendations for male carriers are not described.

3.1.8. French National Cancer Institute Guidelines. The guidelines on early breast and ovarian cancer detection and risk-reducing strategies for female $B R C A$ carriers from the French National Cancer Institute (Institut National du Cancer, INCa) were published in 2017 [44]. In female carriers $<30$ years of age, annual clinical breast exam is recommended, with the addition of imaging only in case of early familial antecedents. Between age 30 and 65, annual synchronous MRI and mammogram are recommended with the addition of ultrasonography on indication, six-monthly alternating with a clinical breast exam. Specific guidance on imaging technique (e.g., single oblique incidence in conjunction with breast MRI) and radiologist requirements are described. For female carriers above age 65, annual mammography (double incidence) is recommended. Regarding the upper age limit, comorbidities and life expectancy have to be considered.

3.1.9. National Breast Cancer Council Netherlands. The breast cancer surveillance guidance for BRCA mutation carriers from the Dutch national breast cancer guidelines (Nationaal Borstkanker Overleg Nederland, NABON) were last revised in 2017 [45]. Annual clinical breast examination is recommended between 25 and 75 years. Interestingly, regarding breast imaging guidelines, a differentiation between $B R C A 1$ and $B R C A 2$ is made. For $B R C A 1$ carriers, only annual breast MRI is advised between 25 and 40 years. Between age 40 and 60, annual MRI and biannual mammogram is recommended. For BRCA2 carriers, annual breast MRI is recommended from age 25 onwards, with the addition of annual mammogram starting at age 30 . Between age 60 and age 75 annual mammogram is recommended, where in case of high breast density annual imaging with alternating MRI and mammogram should be considered, both in BRCA1 and BRCA2 carriers. 
3.1.10. Belgian Society of Human Genetics. The Belgian guidelines for managing hereditary breast and ovarian cancer were developed in 2019 within the working group oncogenetics from the College of Genetics and Rare disease and the Belgian Society of Human Genetics (BeSHG) and are endorsed by the hereditary cancer task force of the Belgian society of Medical Oncology (BSMO) [46]. For female BRCA carriers, clinical breast examination is recommended every 6 months from age 25 onwards. Between age 25-35, annual breast MRI is advised. At age 30 a first baseline mammogram is recommended. In case microcalcifications are present as a possible reflection of in situ carcinoma, yearly mammogram (+/- ultrasound when indicated by the radiologist) should be recommended from age 30 onwards in situations where no treatment is indicated yet, whereas in the absence of these findings annual mammogram can be considered from 30 onwards, but is only routinely recommended from age 35 . Between 35 and 65 years, both breast MRI and mammogram (+/- ultrasound) are recommended, alternating every 6 months. Between age 65 and 75, annual mammography is recommended, and MRI should only be considered in case of residual dense breast tissue or other findings on breast imaging where added value of MRI could be expected. For women $>75$ years, a biannual mammogram should be considered. With regard to male breast cancer, routine screening is not recommended for $B R C A 1$, while for BRCA2 annual clinical exam can be considered starting from age 40 onwards.

3.2. Ovarian, Fallopian Tube, and Primary Peritoneal Cancer. Carriers of a pathogenic $B R C A$ mutation are at high risk for epithelial ovarian, fallopian tube and primary peritoneal cancer, with a cumulative risk at 80 years of $44 \%$ for $B R C A 1$ and $17 \%$ for BRCA2 [10,47]. Ovarian cancer incidence increases slowly from approximately 35 years onwards in patients with BRCA1-and from around 50 years onwards in BRCA2-mutations. In contrast to breast cancer where both prophylactic mastectomy and medical surveillance are reasonable, outcomes of epithelial ovarian cancer are poor and there are major limitations regarding early detection. Risk-reducing salpingo-oophorectomy (RRSO) provides an important reduction in ovarian and breast cancer risks and related mortality; however, the latter is less clearly demonstrated for BRCA2 [48-50]. Therefore, all female carriers with (likely) pathogenic $B R C A$ variants should be recommended to undergo risk-reducing surgery of the fallopian tubes and ovaries after completion of childbearing [37]. With regard to the timing of surgery, quality of life and ageadjusted ovarian cancer risk should be considered. In $B R C A 1$ carriers, RRSO is usually advised between the age of 35 to 40 , after completion of childbearing. Because later onset of disease in BRCA2 mutation carriers, RRSO can be advised between the age of 40-50, however some guidelines still use the 35 lower age limit for RRSO recommendation for BRCA2 [37, 46, 51]. Although there is some evidence regarding the safety of interval salpingectomy (with retention of the ovaries) as initial procedure with the goal to decline or delay menopause initiation, more data are needed before this can be routinely recommended. Clinical trials investigating the safety of this procedure are currently ongoing (e.g., NCT02321228) [52]. Due to this strong recommendation for risk-reducing surgery, ovarian cancer surveillance only is applicable in patients who refuse or have not yet undergone RRSO. Primary peritoneal carcinoma after RRSO has been reported mainly in $B R C A 1$ carriers but remains rare. Moreover this stays a controversial entity since this could possibly reflect a metastatic lesion arising from serous tubal intraepithelial carcinoma (STIC), which is a precursor lesion of high-grade serous ovarian cancer $[53,54]$. Therefore, the risk of primary peritoneal carcinoma in $B R C A$ carriers is not discussed in the majority of secondary prevention guidelines. Adequate pathological examination of RRSO specimens by the 'standardized sectioning and extensively examining the fimbriated end' protocol (SEE-FIM) is necessary in order to detect the presence of precancerous lesions in the fallopian tube, e.g., serous tubal intraepithelial carcinomas [55]. These lesions warrant further staging, as they were correlated with metastatic potential in sporadic ovarian cancer [54].

3.2.1. European Society for Medical Oncology Guidelines. The clinical practice guidelines for cancer prevention and screening in BRCA mutation carriers from ESMO emphasize the technical limitations for early detection of ovarian cancer and that there are no data proving that screening for ovarian cancer in BRCA carriers reduces mortality [36]. There are some promising results with serial CA125 screening, but sufficient data are unavailable [56]. Before RRSO, sixmonthly transvaginal ultrasound and serial measures of serum CA125 could be considered from the age of 30 . The limited data on this approach should be communicated with the patient. After RRSO, surveillance for the residual risk of peritoneal carcinoma is not recommended.

\subsubsection{National Comprehensive Cancer Network Guidelines.} The NCCN clinical practice guidelines in genetic high-risk assessment for breast, ovarian and pancreatic cancer state that transvaginal ultrasound combined with serum CA125 measures for ovarian cancer screening may be considered in $B R C A$ mutation carriers who have not underwent elective RRSO starting at age 30 to 35 . The benefit of this screening is uncertain.

3.2.3. American College of Obstetricians and Gynecologists/ Society of Gynecologic Oncology. The HBOC clinical management guidelines from the ACOG/SGO do not generally recommend routine ovarian cancer screening with measurement of serum CA125 or transvaginal ultrasonography [41]. These screening procedures have not proven to decrease mortality rate or increase survival rate associated with ovarian cancer-specific mortality. Transvaginal ultrasonography or CA125 measures are reasonable options for shortterm surveillance in women at high risk of ovarian cancer, starting at age 30-35 years and continuing until they opt for RRSO. 
3.2.4. American College of Radiology. The publication of the Appropriateness Criteria ${ }^{\circledR}$ for ovarian cancer screening from the American College of Radiology (ACR) dates from 2017 [57]. They state that transvaginal, transabdominal and color Doppler of the ovaries may be appropriate in premenopausal and postmenopausal BRCA carriers, and specify that other imaging techniques are usually not appropriate.

3.2.5. Spanish Society of Medical Oncology. The SEOM clinical guidelines in HBOC advise considering six-monthly transvaginal ultrasound and CA125 measures from the age of 30 in BRCA1/2 mutation carriers until the age of RRSO, as well as for those who have not elected RRSO [42].

3.2.6. French National Cancer Institute Guidelines. The French guidelines by INCa recommend annual clinical pelvic examination as screening for ovarian cancer in BRCA1 and BRCA2 mutation carriers [44]. Starting age is not specified.

3.2.7. National Breast and Ovarian Cancer Council Netherlands. The breast cancer surveillance guidance for $B R C A$ mutation carriers from the NABON specifies that screening for ovarian cancer in BRCA1 and BRCA2 mutation carriers is not recommended [45].

The familial and hereditary ovarian cancer guidelines from the Dutch cancer center (Integraal Kankercentrum Nederland, IKNL) advise counseling carriers on the absence of data that supports effectivity of ovarian screening and recommend not offering ovarian screening to $B R C A$ carriers [58].

3.2.8. Belgian Society of Human Genetics. The Belgian guidelines for managing $\mathrm{HBOC}$ do not recommend screening for ovarian cancer in $B R C A 1$ or $B R C A 2$ mutation carriers [46]. A tailored screening program could be offered from age 40, when the patient refuses RRSO.

3.2.9. Institute of Cancer Research. While the NICE guidelines do not mention the option of ovarian cancer screening, the ICR BRCA mutation carrier guidelines specify that ovarian surveillance is not recommended [40].

3.3. Pancreatic Cancer. The risk of pancreatic adenocarcinoma is increased in BRCA2 mutation carriers, while data for $B R C A 1$ are conflicting $[9,14,59]$. Screening in high-risk patients, like BRCA mutation carriers with familial antecedents, might be beneficial given the high mortality rate of pancreatic adenocarcinoma. Data suggest that screening is able to detect earlier stages of pancreatic cancer that are still curable, in comparison to people who are diagnosed with symptomatic disease [60]. Also in pancreatic cancer, $B R C A$ pathogenic variants have therapeutic implications [6]. Given the well documented correlation between smoking and pancreatic adenocarcinoma, additional counseling for smoking cessation in this regard seems to be an important primary prevention strategy in $B R C A$ mutation carriers $[61,62]$.

3.3.1. European Society for Medical Oncology Guidelines. The ESMO clinical practice guidelines for cancer prevention and screening in $B R C A$ mutation carriers state that annual screening for pancreatic cancer may be considered in BRCA2 mutation carriers [36]. People should be informed about the very limited available data for this approach. There is no consensus about when screening should be initiated, but it is reasonable to start at age 50 or 10 years before the earliest diagnosed case of pancreatic cancer in the family. Screening consists of endoscopic ultrasound (EUS) or MRI/magnetic resonance cholangiopancreatography (MRCP). Trials evaluating the efficacy of screening techniques for pancreatic cancers should be strongly encouraged for BRCA carriers.

3.3.2. National Comprehensive Cancer Network Guidelines. The NCCN clinical practice guideline in genetic high-risk assessment for breast, ovarian, and pancreatic cancer does not recommend pancreatic cancer screening for $B R C A 1$ and $B R C A 2$ mutation carriers in the absence of a close family history of exocrine pancreatic cancer [37]. Pancreatic cancer screening can be considered for individuals with exocrine pancreatic cancer in one or more first- or second-degree relatives from the same side of the family as the identified pathogenic/likely pathogenic BRCA1/2 mutation. Screening starts at the age of 50 , or 10 years younger than the earliest exocrine pancreatic cancer diagnosis in the family. Screening recommendations include annual contrast-enhanced MRI/ MRCP and/or EUS, with consideration of shorter screening interval when worrisome abnormalities are found. The majority of small cystic lesions found on screening will not warrant a biopsy or surgical resection. Before attending screening, people should be informed about the potential limitations to screening, including cost, high incidence of pancreatic abnormalities, and uncertainties about the potential benefits. It is recommended that pancreatic cancer screening should be performed in experienced high-volume centers under research conditions.

3.3.3. Spanish Society of Medical Oncology. The SEOM clinical guidelines in HBOC propose to consider pancreatic cancer surveillance with EUS and MRI in carriers with FDG with pancreatic cancer from the age of 50, or 10 years before the youngest diagnosis in the family [42].

3.3.4. Belgian Society of Human Genetics. The BeSHG guidelines propose to discuss the arguments in favor of and against pancreatic cancer screening with $B R C A 1$ carriers if they have $\geq 1$ first-degree relative with pancreatic cancer and with $B R C A 2$ carriers if they have $\geq 1$ first-degree or $\geq 2$ second-degree relatives with pancreatic cancer [46]. This should preferably be performed in the context of a clinical trial. Regarding the starting age and screening modality, they refer to the recommendation from the International Cancer of the Pancreas Screening Consortium [59]. Recommended 
starting age for BRCA carriers with familial antecedents as described above is 50 years, unless there is a first-degree relative with pancreatic cancer onset $<50$ years. Surveillance for pancreatic cancer should consist of MRI/MRCP and an EUS combined with fasting blood glucose and/or HbAlc. Annual blood sugar tests and imaging are recommended; however, there is no consensus as to whether and how to alternate MRI/MRCP and EUS. Serum CA 19-9 is not routinely recommended.

3.4. Prostate Cancer. The link between an elevated risk of prostate cancer and germline $B R C A$ pathogenic variants has been well established, with the strongest association for BRCA2 [63, 64]. Prostate cancer in germline BRCA2 carriers appears to occur at an earlier age; has a more aggressive phenotype, a higher risk of nodal involvement, and distant metastasis; and is associated with a poor survival outcome in comparison to noncarriers. In advanced castration-resistant prostate cancer, $B R C A$ status can have therapeutic implications regarding the use of platinum agents and PARP inhibitors $[65,66]$.

3.4.1. European Society for Medical Oncology Guidelines. The ESMO clinical practice guidelines state that annual screening for prostate cancer may be considered from age 40 onwards, particularly for BRCA2 mutation carriers [36]. The optimal duration of screening is not specified but should be tailored to an individual's family history of prostate cancer.

3.4.2. National Comprehensive Cancer Network Guidelines. The NCCN clinical practice guidelines in genetic high-risk assessment for breast, ovarian, and pancreatic cancer refer to the NCCN prostate cancer early detection guidelines for prostate cancer screening in $B R C A$ carriers $[37,67]$. Prostate cancer screening in BRCA2 mutation carriers is recommended starting at the age of 40 , whereas in BRCA1 mutation, carriers screening should be considered from the age of 40 onwards. Shared decision making is recommended. In men older than 75 years, prostate cancer screening should be considered in selected patients only. The NCCN prostate cancer early detection guidelines specify yearly screening for PSA. Digital rectal examination (DRE) should not be used as a stand-alone test but may be considered as baseline test and as follow-up exam as it may identify high-grade cancers associated with low serum PSA values. It should be performed in carriers with an elevated serum PSA. Referral for biopsy should be considered if DRE is very suspicious.

3.4.3. Spanish Society of Medical Oncology. The hereditary cancer working group from SEOM recommends prostate cancer screening with annual serum PSA measurements in male $B R C A 2$ carriers starting at age 40 , while this screening approach can also be offered to BRCA1 carriers [42].

3.4.4. Belgian Society of Human Genetics. The Belgian Society of Human Genetics recommends annual prostate cancer screening with serum PSA and DRE for male BRCA1 and BRCA2 mutation carriers from the age of 40 onwards [46].

3.5. Colorectal and Gastric Cancer. Data about a possible relationship between gastric and colorectal cancer (CRC) and germline $B R C A$ pathogenic variants are conflicting. A large prospective study on 7015 women with $B R C A$ alterations showed a significant increased risk for CRC in women younger than 50 years with a $B R C A 1$ mutation, but not in older $B R C A 1$ carriers or in BRCA2 carriers [68, 69]. A systematic review and meta-analysis confirmed the differential effect between BRCA1 and BRCA2 (odds ratio [OR] 1.49 [95\% CI 1.19-1.85] for BRCA1, not significant [OR 1.1; 95\% CI 0.77-1.58] for BRCA2), but could not validate the age effect [19]. Regarding a possible relationship with gastric cancer, there is only weak evidence for a correlation with germline BRCA mutations and gastric cancer; anecdotal findings have not been confirmed in larger series $[8,70,71]$. These recent findings warrant increased attention to familial $\mathrm{CRC}$ and possibly gastric cancer antecedents and the need for individualized surveillance in BRCA carriers. The majority of guidelines do not mention the possible increased risk for digestive tract cancer.

\subsubsection{European Society for Medical Oncology Guidelines.} The ESMO clinical practice guidelines state that the association between $B R C A$ mutation carriers and an elevated risk of colorectal and gastric cancer is weak [36]. Therefore, screening is generally not indicated. Recommendations should be tailored to an individual's familial history.

3.5.2. Spanish Society of Medical Oncology. The clinical guidelines in HBOC of the hereditary cancer working group from SEOM point towards the controversial results on the association of BRCA1/2-mutations and colorectal cancer and towards the possible differences between BRCA1 and $B R C A 2$, but latest version of these guidelines does not mention colorectal cancer surveillance, while specific recommendations for BRCA1 were reported in the previous version $[42,51]$.

3.5.3. Belgian Society of Human Genetics. The BeSHG guidelines for managing $\mathrm{HBOC}$ indicate that $B R C A 1 \mathrm{mu}-$ tation carriers have an increased risk of early-onset colorectal cancer (diagnosis $<50$ years), but the increase is small. Screening for colorectal cancer is not recommended for this elevated risk besides the national population screening program independent of $B R C A$ status which offers biannual fecal blood test between 50 and 74 years of age in absence of familial history [46]. Also here, a possible correlation with elevated gastric cancer risk is not mentioned.

3.6. Endometrial Cancer. Some data suggest a slightly increased risk of endometrial cancer in $B R C A$ carriers, with more evidence for a correlation with $B R C A 1$ and then with 
$B R C A 2$; however, the risk is not clearly defined. Several reports showed that tamoxifen use for previous breast cancer is an important confounding factor in the earlier observed correlations between endometrial cancer and germline $B R C A$ mutations $[72,73]$. A prospective cohort study analyzing the risk of endometrial cancer after RRSO in 1083 $B R C A$ carriers showed no significant increase of endometrial cancer overall, but a higher than expected risk of serous endometrial carcinoma in BRCA1 mutation carriers (however, only 4 cases were described in 453 BRCA1 carriers after a median follow-up of 5.1 years), while the risk for endometrioid endometrial cancer or uterine sarcoma was not increased in this study [20]. Another cohort study on 828 carriers could not confirm the correlation with serous endometrial cancer. Overall, no significant correlation with endometrial cancer was demonstrated, but there was a possible trend for the endometrioid subtype [74]. Based on these findings, some guidelines advise discussing these risk uncertainties and the risks and benefits of concurrent hysterectomy at the time of RRSO in female BRCA1 carriers [37]. However, the majority of guidelines do not recommend considering hysterectomy for the presumed increased risk of endometrial cancer. In female $B R C A$ carriers who have opted for breast surveillance instead of risk-reducing mastectomy, there is more data on safety with regard to breast cancer risk of estrogen-only hormonal substitution compared to combined estrogen-progesterone substitution after RRSO [75]. With regard to endometrial cancer risk, however, estrogen-only substitution is not a safe option when no hysterectomy has been performed, making this an additional factor to be considered in these discussions [76].

3.6.1. European Society for Medical Oncology Guidelines. The ESMO clinical practice guidelines for cancer prevention and screening in $B R C A$ mutation carriers report that the association between $B R C A 1 / B R C A 2$ mutations and an elevated risk of endometrial cancer remains weak [36]. They conclude that screening for and prevention of endometrial cancer are generally not indicated. Recommendations should be tailored to an individual's familial history.

\subsubsection{National Comprehensive Cancer Network Guidelines.} The NCCN guidelines state that there is limited data suggesting there might be a slightly increased risk of serous endometrial cancer among women with a BRCA1 pathogenic or likely pathogenic variant [37]. The clinical significance is unclear. There is no guidance with regard to screening or prevention. Further evaluation of the risk of serous endometrial cancer in the $B R C A$ population needs to be undertaken.

3.6.3. Belgian Society of Human Genetics. Surveillance and prevention of endometrial cancer in BRCA1 mutation carriers are not advised by the Belgian Society of Human Genetics, because the cumulative risk of serous endometrial cancer is less than $5 \%$ at 70 years of age. The risk in BRCA2 mutation carriers is described as equal to a population without germline BRCA pathogenic variants.

3.7. Melanoma. Literature suggests a possible association between germline BRCA2 pathogenic variants and an elevated risk for melanoma. This possible link has been suggested for both cutaneous and ocular melanoma in BRCA2, but data are conflicting and mainly based on small studies at risk for sampling bias $[9,15,21,77]$. Overall there seems to be insufficient evidence for a clear correlation between skin and uveal melanoma and germline $B R C A$ pathogenic variants. However, increased awareness of familial history and preventive measurements in BRCA carriers seems reasonable.

3.7.1. European Society for Medical Oncology Guidelines. The ESMO guidelines demonstrate that there is no evidencebased data with regard to screening for melanoma [36]. They advise considering annual skin and eye examination as screening for melanoma in all BRCA2 carriers. Screening should be tailored to the individual's family history.

\subsubsection{National Comprehensive Cancer Network Guidelines.} The NCCN guidelines of genetic high-risk assessment for breast, ovarian, and pancreatic cancer state that no specific screening guidelines exist for melanoma, but general melanoma risk management with education regarding clinical signs, minimizing UV exposure, and annual full-body skin examination with the addition of an eye exam should be considered for both BRCA1 and BRCA2 mutation carriers with a pathogenic or likely pathogenic mutation [37]. An individualized screening approach based on personal and family history of cancer may be provided.

3.7.3. Spanish Society of Medical Oncology. In the SEOM clinical guidelines in HBOC, screening for melanoma with a skin and eye examination should be considered according to personal and familial risk factors [42]. It is not specified if this applies to BRCA1 and/or BRCA2 mutation carriers.

\section{Discussion}

This review demonstrates that there are major differences in national and international guidelines on early detection of and screening for $B R C A$-related cancers in $B R C A$ carriers. These differences are triggered by temporal evolution in risk assessments, discordances in literature and interpretation, assessment of the advantages and disadvantages of screening, cost-benefit analyses, and absence of high levels of evidence. As the case for cancer screening in the general population, different thresholds and risk/benefit analyses are used by different societies publishing guidelines for HBOC. More harmonized guidelines could be relevant from a clinical perspective, but this is hard to implement at a global level for the reasons stated above. However, harmonization efforts by translation of international guidelines into the local context in regional or national guidelines can be of high 
value to avoid differences in counseling and risk management advice.

In general, guidelines are more concordant for $B R C A$ related cancers in situations where the age-specific risks for this cancer type are more extensively studied, while there is more discordance in other BRCA-related cancers. However, also in breast cancer, there are differences in screening modalities, thresholds, and frequency and duration of screening. The majority of guidelines recommend starting imaging surveillance in female carriers from age 25 onwards and also consider screening for all untested first-degree relatives of $B R C A$ carriers [34, 36, 37]. Only occasionally, a differentiation between $B R C A 1$ and $B R C A 2$ carriers is made with a trend to start later or decrease mammography frequency in BRCA1 compared to BRCA2 carriers, probably based on the possible higher likelihood of microcalcifications as a reflection of in situ carcinoma in ERpositive breast cancers which are enriched in BRCA2 $[42,45]$. However, this differential correlation with in situ carcinoma has not been confirmed [78]. The age of onset of mammographic surveillance varies significantly between the different guidelines as described above. There seems to be a potential of adding digital breast tomosynthesis to the imaging surveillance for breast cancer in some women given the higher sensitivity and specificity compared to routine mammogram and possibly decreasing false positive findings of standard mammography, and some guidelines already describe this option [34,37]. Individualization of starting age based on family history is recommended in the majority of guidelines. In the concept of shared decision making, patient preference is a very important consideration in the discussion of breast cancer surveillance strategies and riskreducing options. There are still a lot of open questions regarding optimal breast cancer screening in $B R C A$ carriers, e.g., recommended surveillance when MRI is not possible/ unavailable, optimal age to discontinue surveillance, value of ultrasonography, and value of alternating versus concomitant imaging when 2 modalities are combined. These and other questions stress the importance of ongoing and future studies.

In contrast to RRSO, there is no evidence that screening for ovarian cancer in $B R C A$ carriers reduces mortality. RRSO should be recommended for all BRCA mutation carriers, with important differences in age recommendations for RRSO between different guidelines. Some guidelines consider screening for ovarian cancer in people refusing RRSO. Others also consider surveillance in $B R C A$ carriers before RRSO is performed. If this is considered, it is of utmost importance that patients are informed that there is no proven benefit of screening with serial CA125 measurements and transvaginal ultrasonography.

All the four guidelines that covered pancreatic carcinoma considered screening only in the presence of a positive familial history and after proper counseling of advantages and disadvantages of pancreatic cancer screening. There is no consensus as to whether it should be proposed to BRCA2 carriers only or both, or about the screening modality or the age when screening should start. Because it is unknown if pancreatic cancer screening impacts overall survival, it is preferred to perform pancreatic cancer screening in the context of clinical trials and in high-volume centers.

The link between $B R C A$ mutation carriers and prostate cancer has been well established. Screening is recommended by the NCCN, SEOM, and BeSHG guidelines, while the ESMO guidelines consider it $[36,37,46,51]$. Differentiation is made between $B R C A 1$ and $B R C A 2$ carriers based on the higher penetrance of prostate cancer in male $B R C A 2$ carriers [67]. Most guidelines recommend PSA and DRE as screening methods, but optimal duration is not properly addressed.

Because there is less evidence about an association between $B R C A$ pathogenic variants and colorectal, endometrial, skin, and gastric cancer, the majority of guidelines do not recommend systematic screening. Raised awareness and careful incorporation of familial history to individualize primary and secondary prevention for these cancer types seem appropriate. Further investigation of these cancer risks in BRCA carriers and evaluation of surveillance methods in clinical trials are warranted.

\section{Conclusion}

There are major differences between available guidelines for cancer surveillance in germline BRCA mutation carriers. Optimal surveillance strategies depend on accurate agespecific cancer risk estimates, which are reliably estimated for breast and ovarian cancer but not for other BRCA-related cancers. Up-to-date national or international consensus guidelines are of utmost importance to harmonize counseling and proposed surveillance strategies for BRCA1/2 carriers. Improving awareness of carriers and primary care physicians together with shared decision making is a key aspect of cancer surveillance in BRCA carriers. Possible benefits of screening and risk-reducing strategies should always be discussed in combination with possible risks and limitations of these surveillance strategies.

\section{Conflicts of Interest}

RdP receives honoraria for advisory board for Astra Zeneca. ML has the advisory role for Roche and receives speaker honoraria from Theramex, Takeda, Roche, and Lilly. AT has the advisory role for Lilly and Roche. CVO, MK, and RP authorize the advisory role for the Belgian Society of Human Genetics guidelines. WE receives honoraria for consulting and speaker fees from Janssen, Astellas, and Bayer. He is a senior clinical researcher of FWO Flanders. HW receives consulting fees and honoraria from Abbvie, Amgen, Ariez International, Astra Zeneca, Biocartes, Celldex Therapeutics, DNA Prime, Janssen-CILAG, Lilly, Novartis, ORION Corporation, Pfizer, The Planning Shop, PUMA Biotechnology, Roche, Sirtex, TRM Oncology, and Vifor Pharma. $\mathrm{He}$ also receives travel support from Roche, Pfizer, Nippon Travel Agency, Congress Care, DNA Prime, and Global Teamwork. PN receives honoraria for consultancy and/or advisory roles from Pfizer, Novartis, Eli Lilly, and Roche. He receives research funding from Kom op Tegen Kanker. RdP, ED, GVB, and EL are the co-authors of the Belgian Society of 
Human Genetics guidelines. KP has the advisory/consultancy role for Astra Zeneca, Eli Lilly, Novartis, Pfizer, Pierre Fabre, Roche, and Vifor Pharma. He also receives speaker fees for Eli Lilly, Mundi Pharma, Novartis, Pfizer, and Roche and receives research funding from Sanofi. He obtains travel support from Astra Zeneca, Novartis, Pfizer, Pharma Mar, and Roche. The other authors declare that there are no conflicts of interest.

\section{References}

[1] A. C. Antoniou, P. D. P. Pharoah, G. McMullan et al., "A comprehensive model for familial breast cancer incorporating BRCA1, BRCA2 and other genes," British Journal of Cancer, vol. 86, no. 1, pp. 76-83, 2002.

[2] K. Manickam, A. H. Buchanan, M. L. B. Schwartz et al., "Exome sequencing-based screening for BRCA1/2 expected pathogenic variants among adult biobank participants," JAMA Network Open, vol. 1, no. 5, Article ID e182140, 2018.

[3] K. A. Metcalfe, A. Poll, R. Royer et al., "Screening for founder mutations in BRCA1 and BRCA2 in unselected Jewish women," Journal of Clinical Oncology, vol. 28, no. 3, pp. 387-391, 2010.

[4] K. Poll, "Abstract P6-08-03: germline mutational landscape in 5422 individuals at risk for hereditary breast and ovarian cancer who underwent multi-gene panel testing," Cancer Research, vol. 80, pp. P6-P08, 2020.

[5] M. E. Robson, N. Tung, P. Conte et al., "OlympiAD final overall survival and tolerability results: olaparib versus chemotherapy treatment of physician's choice in patients with a germline BRCA mutation and HER2-negative metastatic breast cancer," Annals of Oncology, vol. 30, no. 4, pp. 558-566, 2019.

[6] T. Tung, P. Hammel, M. Reni et al., "Maintenance olaparib for germline BRCA-mutated metastatic pancreatic cancer," New England Journal of Medicine, vol. 381, no. 4, pp. 317-327, 2019.

[7] S. Hammel, O. Pagani, A. H. Partridge et al., "ESO-ESMO 3rd international consensus guidelines for breast cancer in young women (BCY3)," The Breast, vol. 35, pp. 203-217, 2017.

[8] H. Kim, D. H. Choi, W. Park et al., "The association between non-breast and ovary cancers and BRCA mutation in firstand second-degree relatives of high-risk breast cancer patients: a large-scale study of Koreans," Hereditary Cancer in Clinical Practice, vol. 17, no. 1, 2019.

[9] J. Mersch, M. A. Jackson, M. Park et al., "Cancers associated with BRCA1 and BRCA2 mutations other than breast and ovarian," Cancer, vol. 121, no. 2, pp. 269-275, 2015.

[10] K. B. Jackson, J. L. Hopper, D. R. Barnes et al., "Risks of breast, ovarian, and contralateral breast cancer for BRCA1 and BRCA2 mutation carriers," JAMA, vol. 317, no. 23, pp. 2402-2416, 2017.

[11] N. Howlader, A. M. Noone, M. Krapcho et al., SEER Cancer Statistics Review, 1975-2016, National Cancer Institute, Bethesda, MD, USA, 2018, https://seer.cancer.gov/csr/1975_ 2016/.

[12] Y. C. Tai, S. Domchek, G. Parmigiani, and S. Chen, "Breast cancer risk among male BRCA1 and BRCA2 mutation carriers NIH public access," Journal of the National Cancer Institute, vol. 99, no. 23, pp. 1811-1814, 2007.

[13] D. G. R. Evans, I. Susnerwala, J. Dawson, E. Woodward, E. R. Maher, and F. Lalloo, "Risk of breast cancer in male
BRCA2 carriers," Journal of Medical Genetics, vol. 47, no. 10, pp. 710-711, 2010.

[14] J. Iqbal, A. Ragone, J. Lubinski et al., "The incidence of pancreatic cancer in BRCA1 and BRCA2 mutation carriers," British Journal of Cancer, vol. 107, no. 12, pp. 2005-2009, 2012.

[15] A. Moran, C. O’Hara, S. Khan et al., "Risk of cancer other than breast or ovarian in individuals with BRCA1 and BRCA2 mutations," Familial Cancer, vol. 11, pp. 235-242, 2012.

[16] N. Petrucelli, M. B. Daly, and T. Pal, BRCA1-and BRCA2Associated Hereditary Breast and Ovarian Cancer, University of Washington, Seattle, WA, USA, 1993.

[17] D. Leongamornlert, N. Mahmud, M. Tymrakiewicz et al., "Germline BRCA1 mutations increase prostate cancer risk," British Journal of Cancer, vol. 106, no. 10, pp. 1697-1701, 2012.

[18] Z. Kote-Jarai, M. Tymrakiewicz, E. Castro et al., "BRCA2 is a moderate penetrance gene contributing to young-onset prostate cancer: implications for genetic testing in prostate cancer patients," British Journal of Cancer, vol. 105, no. 8, pp. 1230-1234, 2011.

[19] M. Oh, A. McBride, S. Yun et al., "BRCA1 and BRCA2 gene mutations and colorectal cancer risk: systematic review and meta-analysis," Journal of the National Cancer Institute, vol. 110, no. 11, pp. 1178-1189, 2018.

[20] C. A. Shu, M. C. Pike, A. R. Jotwani et al., "Uterine cancer after risk-reducing salpingo-oophorectomy without hysterectomy in women with BRCA Mutations," JAMA Oncology, vol. 2, pp. 1434-1440, 2016.

[21] P. V. Gumaste, "Skin cancer risk in BRCA1/2 mutation carriers," British Journal of Dermatology, vol. 172, pp. 14981506, 2015.

[22] M.-C. King, "Tamoxifen and breast cancer incidence among women with inherited mutations in BRCA1 and BRCA2," JAMA, vol. 286, no. 18, pp. 2251-2256, 2001.

[23] K. A. Phillips, R. L. Milne, M. A. Rookus et al., "Tamoxifen and risk of contralateral breast cancer for BRCA1 and BRCA2 mutation carriers," Journal of Clinical Oncology: Official Journal of the American Society of Clinical Oncology, vol. 31, no. 25, pp. 3091-3099, 2013.

[24] L. Hopper, H. Ramos, J. B. Loureiro, J. Calheiros, and L. Saraiva, "BRCA1/P53: two strengths in cancer chemoprevention," Biochimica et Biophysica Acta-Reviews on Cancer, vol. 1873, no. 1, 2020.

[25] S. M. Domchek, "Association of risk-reducing surgery in BRCA1 or BRCA2 mutation carriers with cancer risk and mortality," JAMA, vol. 304, no. 9, pp. 967-975, 2010.

[26] B. A. M. Heemskerk-Gerritsen, M. B. E. Menke-Pluijmers, A. Jager et al., "Substantial breast cancer risk reduction and potential survival benefit after bilateral mastectomy when compared with surveillance in healthy BRCA1 and BRCA2 mutation carriers: a prospective analysis," Annals of Oncology, vol. 24, no. 8, pp. 2029-2035, 2013.

[27] R. Alaofi, M. Nassif, and M. Al-Hajeili, "Prophylactic mastectomy for the prevention of breast cancer: review of the literature," Avicenna Journal of Medicine, vol. 8, no. 3, p. 67, 2018.

[28] B. A. M. Heemskerk-Gerritsen, A. Jager, L. B. Koppert et al., "Survival after bilateral risk-reducing mastectomy in healthy BRCA1 and BRCA2 mutation carriers," Breast Cancer Research and Treatment, vol. 177, no. 3, pp. 723-733, 2019.

[29] P. Neven, K. Punie, H. Wildiers et al., "Risk-reducing mastectomy in BRCA carriers: survival is not the issue," Breast Cancer Research and Treatment, vol. 179, no. 1, pp. 251-252, 2020. 
[30] F. Cardoso, J. M. S. Bartlett, L. Slaets et al., "Characterization of male breast cancer: results of the EORTC 10085/TBCRC/ BIG/NABCG international male breast cancer program," Annals of Oncology, vol. 29, no. 2, pp. 405-417, 2018.

[31] U. Bick, C. Engel, B. Krug et al., "High-risk breast cancer surveillance with MRI: 10-year experience from the German consortium for hereditary breast and ovarian cancer," Breast Cancer Research and Treatment, vol. 175, no. 1, pp. 217-228, 2019.

[32] D. G. Evans, E. F. Harkness, A. Howell et al., "Intensive breast screening in BRCA2 mutation carriers is associated with reduced breast cancer specific and all cause mortality," $\mathrm{He}$ reditary Cancer in Clinical Practice, vol. 14, no. 1, p. 8, 2016.

[33] A. Pijpe, N. Andrieu, D. F. Easton et al., "Exposure to diagnostic radiation and risk of breast cancer among carriers of BRCA1/2 mutations: retrospective cohort study (GENERAD-RISK)," BMJ, vol. 345, no. 2, Article ID e5660, 2012.

[34] M. B. Mainiero, L. Moy, P. Baron et al., "ACR appropriateness criteria ${ }^{\circledR}$ breast cancer screening," Journal of the American College of Radiology, vol. 14, no. 11, pp. S383-S390, 2017.

[35] C. E. Comstock, C. Gatsonis, G. M. Newstead et al., "Comparison of abbreviated breast MRI vs digital breast tomosynthesis for breast cancer detection among women with dense breasts undergoing screening," JAMA, vol. 323, no. 8 , p. 746, 2020.

[36] S. Paluch-Shimon, F. Cardoso, C. Sessa et al., "Prevention and screening in BRCA mutation carriers and other breast/ ovarian hereditary cancer syndromes: ESMO clinical practice guidelines for cancer prevention and screening," Annals of Oncology, vol. 27, pp. v103-v110, 2016.

[37] M. B. Daly, C. Klein, H. L. Pederson et al., NCCN Guidelines: Genetic/Familial High-Risk Assessment: Breast, Ovarian, and Pancreatic, NCCN, Plymouth Meeting, PA, USA, 2019, https:// www.nccn.org/professionals/physician_gls/pdf/genetics_bop.pdf.

[38] NICE Guidelines Committee, Familial Breast Cancer: Classification, Care and Managing Breast Cancer and Related Risks in People with a Family History of Breast Cancer, National Institute for Health and Care Excellence, London, UK, 2019, https://www.nice.org.uk/guidance/cg164.

[39] London Cancer Alliance Breast Pathway Group, London Cancer Alliance Breast Cancer Clinical Guidelines, 2013, https://www. england.nhs.uk/mids-east/wp-content/uploads/sites/7/2018/02/ guidelines-for-the-management-of-breast-cancer-vl.pdf.

[40] Cancer Genetics Clinical Academic Unit at The Royal Marsden and The Institute of Cancer Research, Cancer Genetic Clinical Protocols, Protocol 3: BRCA Mutation Carrier Guidelines, 2015, https://diijoxngr27nfi.cloudfront.net/research-divisions/protocol3-brca-mutation-carrier-20150209-v4.pdf?sfvrsn=5e7f6f69_2.

[41] K. Kuba and P. S. Bernstein, "ACOG practice bulletin No. 188,” Obstetrics \& Gynecology, vol. 131, no. 6, pp. 1163-1164, 2018.

[42] S. González-Santiago, T. Ramón y Cajal, E. Aguirre et al., "SEOM clinical guidelines in hereditary breast and ovarian cancer (2019)," Clinical and Translational Oncology, vol. 22, no. 2, pp. 193-200, 2020.

[43] N. Ditsch, "Breast cancer risk and prevention. Guidelines of the arbeitsgemeinschaft gynäkologische onkologie (AGO)," 2019, https://www.ago-online.de/fileadmin/ago-online/downloads/_ leitlinien/2019/PDF_EN/2019E_02_Breast_Cancer_Risk_and_ Prevention.pdf.

[44] C. Nogues, "Institut National du Cancer (INCa) Recommandations et référentiels pour femmes porteuses d' une mutation de BRCA1 ou BRCA2. Détection précoce du cancer du sein et des annexes et stratégies de réduction du risque,"
2017, https://www.e-cancer.fr/Expertises-et-publications/ Catalogue-des-publications/Femmes-porteuses-d-unemutation-de-BRCA1-ou-BRCA2-Detection-precoce-ducancer-du-sein-et-des-annexes-et-strategies-de-reductiondu-risque.

[45] Dutch National Breast Cancer Council Guidelines Committee, Dutch National Breast Cancer Council Guidelines (NABON), Screening Outside the Population Screening, 2017, https://www.oncoline.nl/richtlijn/item/index.php?pagina=/ richtlijn/item/pagina.php\&richtlijn_id=1097.

[46] Belgian HBOC Working Group, "Belgian guidelines for managing hereditary breast and ovarian cancer," 2019, https:// www.college-genetics.be/assets/recommandations/fr/guidelines/ Clinical_guidelines_HBOC_management_2019_ENG.pdf.

[47] D. A. Levine, P. A. Argenta, C. J. Yee et al., "Fallopian tube and primary peritoneal carcinomas associated with BRCA mutations," Journal of Clinical Oncology, vol. 21, no. 22, pp. 4222-4227, 2003.

[48] N. D. Kauff, S. M. Domchek, T. M. Friebel et al., "Risk-reducing salpingo-oophorectomy for the prevention of BRCA1and BRCA2-associated breast and gynecologic cancer: a multicenter, prospective study," Journal of Clinical Oncology, vol. 26, no. 8, pp. 1331-1337, 2008.

[49] T. R. Rebbeck, N. D. Kauff, and S. M. Domchek, "Metaanalysis of risk reduction estimates associated with risk-reducing salpingo-oophorectomy in BRCA1 or BRCA2 mutation carriers," JNCI Journal of the National Cancer Institute, vol. 101, no. 2, pp. 80-87, 2009.

[50] G. U. Eleje, A. C. Eke, I. U. Ezebialu, J. I. Ikechebelu, E. O. Ugwu, and O. O. Okonkwo, "Risk-reducing bilateral salpingo-oophorectomy in women with BRCA1 or BRCA2 mutations," Cochrane Database of Systematic Reviews, vol. 8, no. 8, Article ID CD012464, 2018.

[51] G. Llort, I. Chirivella, R. Morales et al., "SEOM clinical guidelines in hereditary breast and ovarian cancer," Clinical and Translational Oncology, vol. 17, no. 12, pp. 956-961, 2015.

[52] F. Gaba, J. Piek, U. Menon, and R. Manchanda, "Risk-reducing early salpingectomy and delayed oophorectomy as a two-staged alternative for primary prevention of ovarian cancer in women at increased risk: a commentary," BJOG: An International Journal of Obstetrics and Gynaecology, vol. 126, no. 7, pp. 831-839, 2019.

[53] M. G. Harmsen, J. M. J. Piek, J. Bulten et al., "Peritoneal carcinomatosis after risk-reducing surgery in BRCA1/2 mutation carriers," Cancer, vol. 124, no. 5, pp. 952-959, 2018.

[54] M. A. Eckert, S. Pan, K. M. Hernandez et al., "Genomics of ovarian cancer progression reveals diverse metastatic trajectories including intraepithelial metastasis to the fallopian tube," Cancer Discovery, vol. 6, no. 12, pp. 1342-1351, 2016.

[55] F. Medeiros, M. G. Muto, Y. Lee et al., "The tubal fimbria is a preferred site for early adenocarcinoma in women with familial ovarian cancer syndrome," The American Journal of Surgical Pathology, vol. 30, no. 2, pp. 230-236, 2006.

[56] U. Menon, A. Ryan, J. Kalsi et al., "Risk algorithm using serial biomarker measurements doubles the number of screen-detected cancers compared with a single-threshold rule in the United Kingdom collaborative trial of ovarian cancer screening," Journal of Clinical Oncology, vol. 33, no. 18, pp. 2062-2071, 2015.

[57] P. V. Pandharipande, K. P. Lowry, C. Reinhold et al., "ACR appropriateness criteria ${ }^{\circledR}$ ovarian cancer screening," Journal of the American College of Radiology, vol. 14, no. 11, pp. S490-S499, 2017. 
[58] Guidelines Commission Gynecological Oncology, "Erfelijk en familiair ovariumcarcinoom, guidelines integraal kankercentrum Nederland (IKNL)," 2015, https://www.oncoline.nl/ erfelijk-en-familiair-ovariumcarcinoom.

[59] M. Goggins, K. A. Overbeek, R. Brand et al., "Management of patients with increased risk for familial pancreatic cancer: updated recommendations from the international cancer of the pancreas screening (CAPS) consortium," Gut, vol. 69, no. 1, pp. 7-17, 2020.

[60] M. I. Canto, J. A. Almario, R. D. Schulick et al., "Risk of neoplastic progression in individuals at high risk for pancreatic cancer undergoing long-term surveillance," Gastroenterology, vol. 155, no. 3, pp. 740-751, 2018.

[61] S. O. Antwi, S. E. Fagan, K. G. Chaffee et al., "Risk of different cancers among first-degree relatives of pancreatic cancer patients: influence of probands' susceptibility gene mutation status," Journal of the National Cancer Institute, vol. 111, no. 3, pp. 264-271, 2019.

[62] C. Bosetti, "Cigarette smoking and pancreatic cancer: an analysis from the international pancreatic cancer case-control consortium (PANC4)," Annals of Oncology, vol. 23, no. 10, p. $2773,2012$.

[63] V. N. Giri, K. E. Knudsen, W. K. Kelly et al., "Role of genetic testing for inherited prostate cancer risk: philadelphia prostate cancer consensus conference 2017," Journal of Clinical Oncology, vol. 36, no. 4, pp. 414-424, 2018.

[64] J. T. Zhen, J. Syed, and K. A. Nguyen, "Genetic testing for hereditary prostate cancer: current status and limitations," Cancer, vol. 124, no. 5, pp. 3105-3117, 2018.

[65] B. Kaufman, R. Shapira-Frommer, R. K. Schmutzler et al., "Olaparib monotherapy in patients with advanced cancer and a germline BRCA1/2 mutation," Journal of Clinical Oncology, vol. 33, no. 3, pp. 244-250, 2015.

[66] M. Hussain, J. Mateo, K. Fizazi et al., "PROfound: phase III study of olaparib versus enzalutamide or abiraterone for metastatic castration-resistant prostate cancer (mCRPC) with homologous recombination repair (HRR) gene alterations," Annals of Oncology, vol. 30, no. 5, p. v881, 2019.

[67] P. R. Carroll, "NCCN clinical practice guidelines in oncology. Prostate cancer: early detection," 2019, https://www.nccn.org/ professionals/physician_gls/default.aspx\#prostate_detection.

[68] C. M. Phelan, J. Iqbal, H. T. Lynch et al., "Incidence of colorectal cancer in BRCA1 and BRCA2 mutation carriers: results from a follow-up study," British Journal of Cancer, vol. 110, no. 2, pp. 530-534, 2014.

[69] V. Sopik, C. Phelan, C. Cybulski, and S. A. Narod, "BRCA1 and BRCA2 mutations and the risk for colorectal cancer," Clinical Genetics, vol. 87, no. 5, pp. 411-418, 2015.

[70] H. Ichikawa, T. Wakai, M. Nagahashi et al., "Pathogenic germline BRCA1/2 mutations and familial predisposition to gastric cancer," JCO Precision Oncology, no. 2, pp. 1-8, 2018.

[71] B. Friedenson, "BRCA1 and BRCA2 pathways and the risk of cancers other than breast or ovarian," Medscape General Medicine, vol. 7, no. 2, p. 60, 2005.

[72] M. E. Beiner, A. Finch, B. Rosen et al., "The risk of endometrial cancer in women with BRCA1 and BRCA2 mutations. A prospective study," Gynecologic Oncology, vol. 104, no. 1, pp. 7-10, 2007.

[73] Y. Segev, J. Iqbal, J. Lubinski et al., "The incidence of endometrial cancer in women with BRCA1 and BRCA2 mutations: an international prospective cohort study," Gynecologic Oncology, vol. 130, no. 1, pp. 127-131, 2013.
[74] Y. C. Lee, R. L. Milne, S. Lheureux et al., "Risk of uterine cancer for BRCA1 and BRCA2 mutation carriers," European Journal of Cancer, vol. 84, pp. 114-120, 2017.

[75] J. Kotsopoulos, J. Gronwald, B. Y. Karlan et al., "Hormone replacement therapy after oophorectomy and breast cancer risk among BRCA1 mutation carriers," JAMA Oncology, vol. 4, no. 8, pp. 1059-1065, 2018.

[76] R. T. Chlebowski, T. E. Rohan, J. E. Manson et al., "Breast cancer after use of estrogen plus progestin and estrogen alone: analyses of data from 2 women's health initiative randomized clinical trials," JAMA Oncology, vol. 1, no. 3, pp. 296-305, 2015.

[77] P. A. Johansson, V. Nathan, L. M. Bourke et al., "Evaluation of the contribution of germline variants in BRCA1 and BRCA2 to uveal and cutaneous melanoma," Melanoma Research, vol. 29, no. 5, pp. 483-490, 2019.

[78] R. L. Yang, H. L. Graves, P. J. Zhang et al., "Characteristics of ductal carcinoma in situ found in BRCA1 and BRCA2 mutation carriers," Journal of the American College of Surgeons, vol. 215, no. 3, p. S126, 2012. 